Document downloaded from:

http://hdl.handle.net/10251/84780

This paper must be cited as:

Singh, S.; Gupta, D.; Martínez Molada, E.; Hueso Pagoaga, JL. (2016). Semilocal and local convergence of a fifth order iteration with Frechet derivative satisfying Holder condition. Applied Mathematics and Computation. 276:266-277. doi:10.1016/j.amc.2015.11.062.

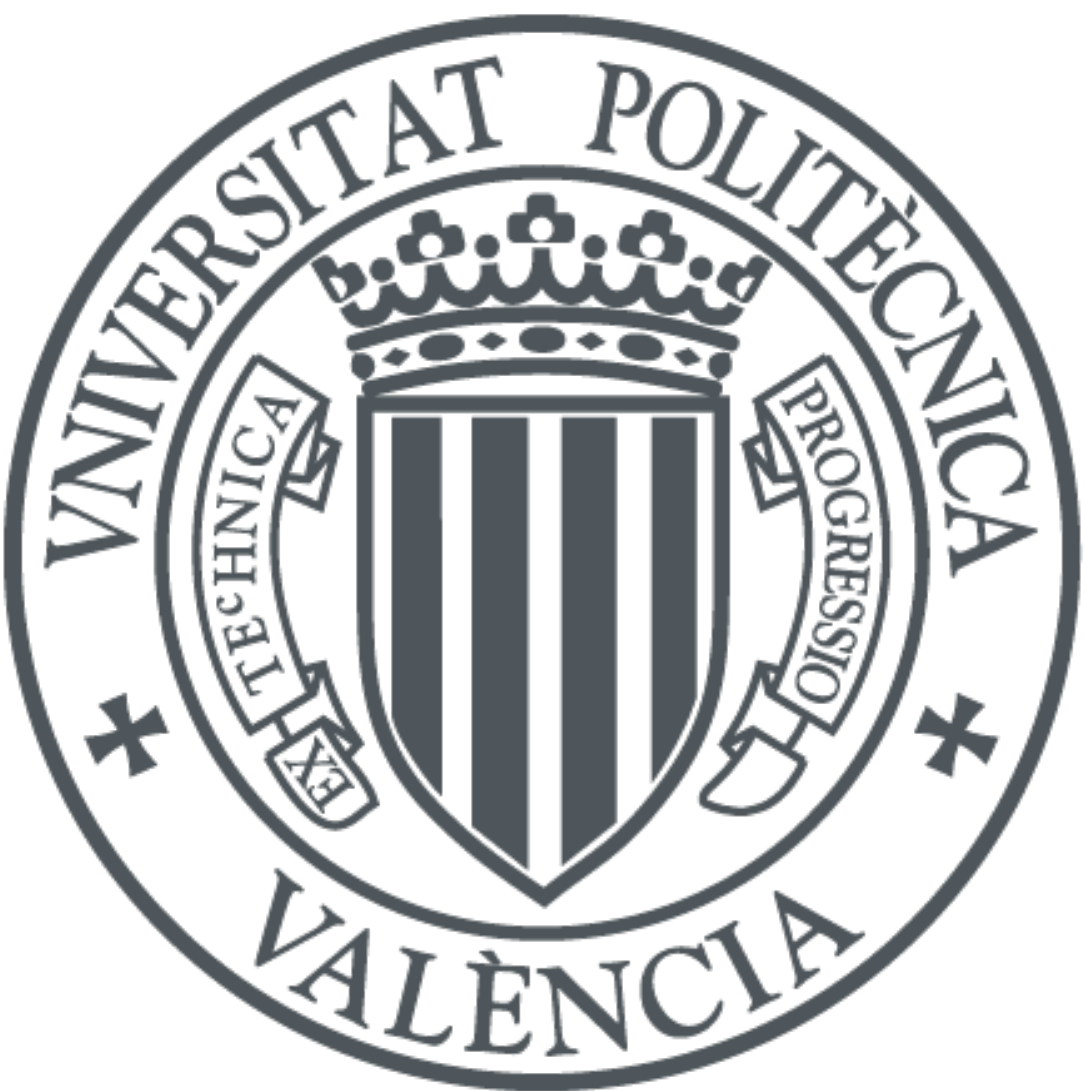

The final publication is available at

http://doi.org/10.1016/j.amc.2015.11.062

Copyright Elsevier

Additional Information 


\title{
Semilocal and local convergence of a fifth order iteration with Fréchet derivative satisfying Hölder condition
}

\author{
Sukhjit Singh ${ }^{1}$ and Dharmendra Kumar Gupta ${ }^{2}$ \\ Department of Mathematics, I.I.T. Kharagpur \\ E. Martínez, Instituto de Matemática Pura y Aplicada ${ }^{3}$ and \\ José L. Hueso, Instituto de Matemática Multidisciplinar ${ }^{4}$, \\ Universitat Politècnica de València
}

\begin{abstract}
The semilocal and local convergence in Banach spaces is described for a fifth order iteration for the solutions of nonlinear equations when the Fréchet derivative satisfies the Hölder condition. The Hölder condition generalizes the Lipschtiz condition. The importance of our work lies in the fact that many examples are available which fail to satisfy the Lipschtiz condition but satisfy the Hölder condition. The existence and uniqueness theorem is established with error bounds for the solution. The convergence analysis is finally worked out on different examples and convergence balls for each of them are obtained. These examples include nonlinear Hammerstein and Fredholm integral equations and a boundary value problem. It is found that the larger radius of convergence balls are obtained for all the examples in comparison to existing methods using stronger conditions.
\end{abstract}

Keywords: Nonlinear equations; Local convergence; Semilocal convergence; Banach space; Hammerstein integral equation; Hölder condition; Fréchet derivative.

Mathematical Subject Classification 65H10, 47H99

\section{Introduction}

Nonlinear equations and their methods for solutions in Banach space setting are extensively studied problems in numerical analysis and scientific computing. It is found that many real life problems arising in science and engineering $[3,4]$ are reduced to find solutions of such equations. It is because their mathematical models involve scaler equations, system of equations, differential equations, integral equations, etc. whose solutions require solving thousands of such equations. The dynamical systems represented by differential equations also lead to solve these equations. Generally, iterative methods with convergence analysis including semilocal and local are used for solving these equations. The semilocal convergence $[7,9]$ uses information given at initial point whereas local convergence $[6,8,10]$ uses information around the solution. Another important problem which is to be considered for these iterative methods is the domains of convergence balls. In general, the convergence domain of an iterative method is small and one always tries to enlarge it by considering additional hypothesis.

\footnotetext{
${ }^{1}$ Corresponding* sukhjit@maths.iitkgp.ernet.in

${ }^{2} \mathrm{dkg} @$ maths.iitkgp.ernet.in

3 eumarti@mat.upv.es

4jlhueso@mat.upv.es
} 
Let $S$ and $T$ are Banach spaces. Consider solving nonlinear equations

$$
G(x)=0
$$

where, $G: D \subseteq S \rightarrow T$ be twice Fréchet differentiable in open convex region $D_{0} \subseteq D$ with values in $T$. Here, we consider the semilocal and local convergence of iteration [1] given by

$$
\begin{aligned}
y_{n} & =x_{n}-\Gamma_{n} G\left(x_{n}\right), \\
z_{n} & =y_{n}-5 \Gamma_{n} G\left(y_{n}\right), \\
x_{n+1} & =z_{n}-\frac{1}{5} \Gamma_{n}\left[-16 G\left(y_{n}\right)+G\left(z_{n}\right)\right], n \geq 0
\end{aligned}
$$

where, $\Gamma_{n}=G^{\prime}\left(x_{n}\right)^{-1}$ and $x_{0}$ is the starting point. Let $G^{\prime}$ satisfies the Hölder condition given by $\left\|G^{\prime}(x)-G^{\prime}(y)\right\| \leq K\|x-y\|^{q}, x, y \in D, q \in(0,1]$. It is worth mentioning that higher order convergence requires computation of derivatives of higher order which are very expansive in general. For example, the third order Chebyshev-Halley type methods [16] require evaluation of $G^{\prime \prime}$ which either does not exist or computationally difficult to evaluate. But higher order methods have their importance as in some applications involving stiff system of equations require faster convergence. Also, it is found that many integral equations involve $G^{\prime \prime}$ which is inexpensive and diagonal by blocks[12]. The semilocal convergence using recurrence relations in [19] and the local convergence in [6] are discussed for a family of iterative methods of order three under Lipschitz condition on $G^{\prime}$. Argyros et al. [13] considered parametric Chebyshev-Halley-type methods with multipoints having high order of convergence with $G^{\prime}$ satisfying Lipschitz condition for their local convergence analysis. The convergence of a modified Halleylike method of high convergence order uses Lipschtiz condition in [14]. A study on the local convergence analysis and the dynamics of Chebyshev-Halley-type methods of convergence order at least five free from second derivative under Lipschitz condition is studied in [15]. The semilocal convergence using recurrence relations in [2] and the local convergence in [5] are studied for (1.2) under Lipschitz condition on $G^{\prime}$. It is to be noted that the the Lipschitz condition is a particular case of Hölder condition. Moreover, examples for which $G^{\prime}$ satisfy Hölder condition but fail to satisfy Lipschitz condition can be constructed.

Example 1.1. Consider an integral equation given by

$$
G(x)(s)=x(s)-1-3 \int_{0}^{1} G_{1}(s, u) x(u)^{5 / 4} d u,
$$

with $x(s) \in \mathcal{C}[0,1]$ and $G_{1}(s, u)$ denotes the Green function.

Therefore,

$$
\left\|G^{\prime}(x)-G^{\prime}(y)\right\| \leq \frac{15}{32}\|x-y\|^{1 / 4}
$$

where, $\left\|G_{1}(s, u)\right\|=1 / 8$. Clearly $G^{\prime}$ satisfies Hölder condition for $q=\frac{1}{4}$ where as Lipschitz condition fails.

Example 1.2. Consider the Fredholm integral equation

$$
G(x)(s)=x(s)-f(s)-\lambda \int_{0}^{1} \frac{s}{s+u} x(u)^{1+q} d u,
$$

with $s \in[0,1], q \in(0,1], x, f \in C[0,1]$ and $\lambda$ is a real number. 
Therefore,

$$
\left\|G^{\prime}(x)-G^{\prime}(y)\right\| \leq|\lambda|(1+q) \log 2\|x-y\|^{q}, x, y \in D .
$$

Clearly, Lipschitz condition fails and Hölder condition holds on $G^{\prime}$ for $q \in(0,1)$.

Using recurrence relations, Ezquerro et al. [17] and Parida and Gupta [18] developed the semilocal convergence analysis of Newton-like methods of order three with $G^{\prime}$ and $G^{\prime \prime}$ satisfying the Hölder condition. Recently, Argyros and George [11] established the convergence of deformed Halley method of third order locally under Hölder condition on $G^{\prime}$. It is defined by

$$
\begin{aligned}
y_{n} & =x_{n}-\Gamma_{n} G\left(x_{n}\right), \\
z_{n} & =x_{n}+\alpha \Gamma_{n} G\left(x_{n}\right), \\
L_{n} & =\frac{1}{\lambda} \Gamma_{n}\left[G^{\prime}\left(x_{n}+\lambda\left(z_{n}-x_{n}\right)\right)-G^{\prime}\left(x_{n}\right)\right], \\
x_{n+1} & =y_{n}+\frac{1}{2} L_{n}\left(I-\frac{1}{2} L_{n}\right)^{-1}\left(y_{n}-x_{n}\right), n \geq 0
\end{aligned}
$$

where, $\Gamma_{n}=G^{\prime}\left(x_{n}\right)^{-1}, \lambda \in(0,1], \alpha \in \mathbb{R}$ and $x_{0}$ is the starting point. This method does not require the computation of expensive $G^{\prime \prime}$.

In this paper, the semilocal and local convergence in Banach spaces is described for a fifth order iteration for the solutions of nonlinear equations when the Fréchet derivative satisfies the Hölder condition. The Hölder condition generalizes the Lipschtiz condition. The importance of our work lies in the fact that many examples are available which fail to satisfy the Lipschtiz condition but satisfy the Hölder condition. The existence and uniqueness theorem is established with error bounds for the solution. The convergence analysis is finally worked out on different examples and convergence balls for each of them are obtained. These examples include nonlinear Hammerstein and Fredholm integral equations and a boundary value problem. It is found that the larger radius of convergence balls are obtained for all the examples in comparison to existing methods using stronger conditions.

This paper is arranged in the following manner. Introduction forms section 1 . The semilocal convergence of an iteration of fifth order under Hölder condition on $G^{\prime}$ is established in Banach spaces in section 2. A theorem for the existence and uniqueness for the solution is given. A number of different numerical examples including a nonlinear Hammerstein and Fredholm integral equations are worked out. The local convergence analysis of fifth order iteration is established in section 3. Here also, a theorem for the existence and uniqueness for the solution is given. The efficacy of our work is demonstrated by working out a number of different numerical examples including a nonlinear Hammerstein integral equation. All the numerical examples are worked out by using high level language MATLAB R2012b on an CPU 3.20GHz with 4GB of RAM running on the windows 7 Professional version 2009 Service Pack 1 on Intel(R) core (TM) i5-3470. Finally, conclusions are included in Section 4.

\section{Semilocal convergence}

In this Section, the semilocal convergence analysis on the iterative method (1.2) for solving (1.1) in Banach spaces is established. First of all, some properties of the sequence $\left\{c_{n}\right\}$ used for this purpose are studied. Next recurrence relations are established. An existence and uniqueness theorem for the solution with error bounds is derived. Finally, our approach is validated by solving some numerical examples. 


\subsection{Property of the sequence $\left\{c_{n}\right\}$}

In this subsection, we shall describe the preliminary results for the convergence of method (1.2) for (1.1). Let $\mathcal{L}(T, S), B(v, \rho)$ and $\bar{B}(v, \rho)$ are the set of bounded linear operators from $T$ to $S$, the open and closed ball in $S$ with center $v$ and radius $\rho>0$, respectively. Let $\Gamma_{0}=G^{\prime}\left(x_{0}\right)^{-1} \in \mathcal{L}(T, S)$ exists at $x_{0} \in D$ and the conditions given below are satisfied.

(1) $\left\|\Gamma_{0}\right\| \leq \beta$

(2) $\left\|\Gamma_{0} G\left(x_{0}\right)\right\| \leq \eta$

(3) $\left\|G^{\prime}(x)-G^{\prime}(y)\right\| \leq K\|x-y\|^{q}, x, y \in D, q \in(0,1]$.

Let $c_{0}=K \beta \eta^{q}$, and define a real sequence for $n \in \mathbb{Z}_{+}$given by

$$
c_{n+1}=c_{n} r\left(c_{n}\right)^{1+q} s\left(c_{n}\right)^{q},
$$

where,

$$
\begin{aligned}
& r(x)=\frac{1}{1-x(1+t(x))^{q}}, \\
& s(x)=\left(\frac{x}{1+q}+(x+1) t(x)+\left(\frac{x}{1+q}\right) t(x)^{1+q}\right),
\end{aligned}
$$

and

$$
t(x)=\left(\frac{x}{1+q}+\frac{x^{2}}{(1+q)^{2}}+\frac{5^{q-1} x^{1+q}}{(1+q)^{1+q}}+\frac{5^{q} x^{2+q}}{(1+q)^{2+q}}\right) .
$$

We will now prove the following lemmas to study the properties of the sequence $\left\{c_{n}\right\}$.

Lemma 2.1. Let functions $r(x), s(x)$ and $t(x)$ be defined by (2.2)-(2.4) and $\tau_{0}$ is the minimum root of $v(x)=x(1+t(x))^{q}-1$ in the interval $(0,1)$. Then

(i) $r(x)$ is increasing and $r(x)>1$ for $x \in\left(0, \tau_{0}\right)$,

(ii) $s(x)$ and $t(x)$ are also increasing functions for $x \in\left(0, \tau_{0}\right)$.

Proof. The proof is trivial.

Consider the auxiliary function

$$
w(x)=\left(\frac{x}{1+q}+(x+1) t(x)+\frac{x t(x)^{1+q}}{1+q}\right)^{q}-\left(1-x(1+t(x))^{q}\right)^{1+q}
$$

Clearly, $w(0)<0, w\left(\tau_{0}\right)>0$ and $w^{\prime}(x)>0$. Thus, $w(x)$ is an increasing function and has a root $\tau_{1}$ in $\left(0, \tau_{0}\right)$.

Lemma 2.2. Let $r(x), s(x)$ and $t(x)$ be the functions defined by (2.2)-(2.4) respectively. If $c_{0} \in\left(0, \tau_{1}\right)$, then

(i) $r\left(c_{0}\right)^{1+q} s\left(c_{0}\right)^{q}<1$,

(ii) $r\left(c_{0}\right) s\left(c_{0}\right)<1$,

(iii) The sequence $\left\{c_{n}\right\}$ is decreasing and $c_{n}<\tau_{1}$ for all $n \geq 0$,

(iv) $c_{n}\left(1+t\left(c_{n}\right)\right)^{q}<1$.

Proof. Taking $x=c_{0}$ in $w(x)$, we get $r\left(c_{0}\right)^{1+q} s\left(c_{0}\right)^{q}<1$ for all $c_{0} \in\left(0, \tau_{1}\right)$. Since $r\left(c_{0}\right)>1$, this gives $\left(r\left(c_{0}\right) s\left(c_{0}\right)\right)^{q}<1$, and hence $\left(r\left(c_{0}\right) s\left(c_{0}\right)\right)<1$. (iii) can be proved by using mathematical induction on (2.1). For $n=0$, this gives $c_{1}=c_{0} r\left(c_{0}\right)^{1+q} s\left(c_{0}\right)^{q}<c_{0}$. Assume that $c_{k}<c_{k-1}$ for $k \leq n$. Since $r$ and $s$ are 
increasing functions, we get $c_{n+1}=c_{n} r\left(c_{n}\right)^{1+q} s\left(c_{n}\right)^{q}<c_{n-1} r\left(c_{n}\right)^{1+q} s\left(c_{n}\right)^{q}<c_{n-1} r\left(c_{n-1}\right)^{1+q} s\left(c_{n-1}\right)^{q}=$ $c_{n}$. Hence, the sequence $\left\{c_{n}\right\}$ is decreasing and $c_{n}<\tau_{1}$ for all $n \geq 0$. Since, $c_{n}<c_{n-1}$ and $t(x)$ is an increasing function, we get $c_{n}\left(1+t\left(c_{n}\right)\right)^{q}<c_{n-1}\left(1+t\left(c_{n-1}\right)\right)^{q}<c_{0}\left(1+t\left(c_{0}\right)\right)^{q}<1 \quad \forall c_{0} \in\left(0, \tau_{1}\right)$. This proves (iv) as $c_{n}\left(1+t\left(c_{n}\right)\right)^{q}<1$ for all $n \geq 0$ and $c_{0} \in\left(0, \tau_{1}\right)$.

\subsection{Recurrence relations}

In this subsection, the recurrence relations for (1.2) is established under the assumptions considered in the earlier subsection. Now, $y_{0}$ exits since $\Gamma_{0}$ exists and hence $K\left\|\Gamma_{0}\right\|\left\|y_{0}-x_{0}\right\|^{q} \leq K \beta \eta^{q}=c_{0}$. Taking $n=0$, in (1.2), we get

$$
\begin{aligned}
z_{0}-x_{0} & =y_{0}-x_{0}-5 \Gamma_{0} G\left(y_{0}\right) \\
& =y_{0}-x_{0}-5 \Gamma_{0} \int_{0}^{1}\left(G^{\prime}\left(x_{0}+\theta\left(y_{0}-x_{0}\right)\right)-G^{\prime}\left(x_{0}\right)\right)\left(y_{0}-x_{0}\right) d \theta .
\end{aligned}
$$

Taking norm on both sides, we get $\left\|z_{0}-x_{0}\right\| \leq\left(1+\frac{5 K \beta}{1+q}\left\|y_{0}-x_{0}\right\|^{q}\right)\left\|y_{0}-x_{0}\right\|$. This implies $\left\|z_{0}-x_{0}\right\| \leq$ $\left(1+\frac{5 c_{0}}{1+q}\right)\left\|y_{0}-x_{0}\right\|$. Similarly, $\left\|z_{0}-y_{0}\right\| \leq \frac{5 c_{0}}{1+q}\left\|y_{0}-x_{0}\right\|$. Now

$$
x_{1}-x_{0}=-\Gamma_{0} G\left(x_{0}\right)-\frac{9}{5} \Gamma_{0} G\left(y_{0}\right)-\frac{1}{5} \Gamma_{0} G\left(z_{0}\right) .
$$

Using Taylor's series expansion of $F\left(z_{0}\right)$ and taking norm both sides, we get

$$
\begin{aligned}
\left\|x_{1}-x_{0}\right\| & \leq\left\|\Gamma_{0} G\left(x_{0}\right)\right\|+\frac{4}{5}\left\|\Gamma_{0}\right\| \int_{0}^{1}\left\|G^{\prime}\left(x_{0}+\theta\left(y_{0}-x_{0}\right)\right)-G^{\prime}\left(x_{0}\right)\right\|\left\|y_{0}-x_{0}\right\| d \theta \\
& +\frac{1}{5}\left\|\Gamma_{0}\right\| \int_{0}^{1}\left\|G^{\prime}\left(x_{0}+\theta\left(z_{0}-x_{0}\right)\right)-G^{\prime}\left(x_{0}\right)\right\|\left\|z_{0}-x_{0}\right\| d \theta \\
& \leq\left\|y_{0}-x_{0}\right\|+\frac{4}{5} \frac{K \beta}{1+q}\left\|y_{0}-x_{0}\right\|^{1+q}+\frac{1}{5} \frac{K \beta}{1+q}\left\|z_{0}-x_{0}\right\|^{1+q} .
\end{aligned}
$$

As,

$$
\frac{1}{5} \frac{K \beta}{1+q}\left\|z_{0}-x_{0}\right\|^{1+q} \leq\left(\frac{c_{0}}{5(1+q)}+\frac{c_{0}^{2}}{(1+q)^{2}}+\frac{5^{q-1} c_{0}^{1+q}}{(1+q)^{1+q}}+\frac{5^{q} c_{0}^{2+q}}{(1+q)^{2+q}}\right)\left\|y_{0}-x_{0}\right\|
$$

so, putting (2.6) in (2.5) and simplifying, we get

$$
\left\|x_{1}-x_{0}\right\| \leq\left(1+t\left(c_{0}\right)\right)\left\|y_{0}-x_{0}\right\| .
$$

The inequalities given below are proved using mathematical induction for $n \geq 1$.

(I) $\quad\left\|\Gamma_{n}\right\| \leq r\left(c_{n-1}\right)\left\|\Gamma_{n-1}\right\|$,

(II) $\quad\left\|y_{n}-x_{n}\right\| \leq\left\|r\left(c_{n-1}\right) s\left(c_{n-1}\right)\right\| y_{n-1}-x_{n-1} \|$,

(III) $\quad\left\|z_{n}-y_{n}\right\| \leq \frac{5}{1+q} c_{n}\left\|y_{n-1}-x_{n-1}\right\|$

(IV) $K\left\|\Gamma_{n}\right\|\left\|y_{n}-x_{n}\right\|^{q} \leq c_{n}$,

(V) $\quad\left\|x_{n}-x_{n-1}\right\| \leq\left(1+t\left(c_{n-1}\right)\right)^{q}\left\|y_{n-1}-x_{n-1}\right\|$.

Assume that $x_{1}, y_{1}, z_{1} \in D$ and $c_{0}<\tau_{0}$. We now have

$$
\left\|I-\Gamma_{0} G^{\prime}\left(x_{1}\right)\right\|=\left\|\Gamma_{0}\left(G^{\prime}\left(x_{1}\right)-G^{\prime}\left(x_{0}\right)\right)\right\| \leq\left\|\Gamma_{0}\right\|\left\|G^{\prime}\left(x_{1}\right)-G^{\prime}\left(x_{0}\right)\right\| \leq K \beta\left\|x_{1}-x_{0}\right\|^{q} .
$$

Using (2.7), this gives $\left\|I-\Gamma_{0} G^{\prime}\left(x_{1}\right)\right\| \leq K \beta\left(1+t\left(c_{0}\right)\right)^{q} \eta^{q} \leq c_{0}\left(1+t\left(c_{0}\right)\right)^{q}<1$. From Banach Lemma, we get $\Gamma_{1}=G^{\prime}\left(x_{1}\right)^{-1}$ and

$$
\left\|\Gamma_{1}\right\| \leq \frac{1}{1-c_{0}\left(1+t\left(c_{0}\right)\right)^{q}}\left\|\Gamma_{0}\right\|=r\left(c_{0}\right)\left\|\Gamma_{0}\right\| .
$$


Expanding $G\left(x_{1}\right)$ using Taylor's formula, we get

$$
G\left(x_{1}\right)=G\left(y_{0}\right)+G^{\prime}\left(y_{0}\right)\left(x_{1}-y_{0}\right)+\int_{y_{0}}^{x_{1}}\left(G^{\prime}(x)-G^{\prime}\left(y_{0}\right)\right) d x .
$$

Now,

$$
\begin{aligned}
\left\|G\left(y_{0}\right)\right\| & \leq \frac{K}{1+q}\left\|y_{0}-x_{0}\right\|^{1+q} \\
\left\|G^{\prime}\left(y_{0}\right)\left(x_{1}-y_{0}\right)\right\| & \leq\left(c_{0}+1\right)\left\|\frac{9}{5} G\left(y_{0}\right)+\frac{1}{5} G\left(z_{0}\right)\right\|, \\
& \leq \frac{\left(c_{0}+1\right)}{\beta} t\left(c_{0}\right)\left\|y_{0}-x_{0}\right\|,
\end{aligned}
$$

since

$$
\begin{aligned}
\left\|\frac{9}{5} G\left(y_{0}\right)+\frac{1}{5} G\left(z_{0}\right)\right\| & \leq \frac{1}{\beta}\left(\frac{c_{0}}{1+q}+\frac{c_{0}^{2}}{(1+q)^{2}}+\frac{5^{q-1} c_{0}^{1+q}}{(1+q)^{1+q}}+\frac{5^{q} c_{0}^{2+q}}{(1+q)^{2+q}}\right)\left\|y_{0}-x_{0}\right\|, \\
& \leq \frac{1}{\beta} t\left(c_{0}\right)\left\|y_{0}-x_{0}\right\| .
\end{aligned}
$$

and

$$
\left\|\int_{y_{0}}^{x_{1}}\left(G^{\prime}(x)-G^{\prime}\left(y_{0}\right)\right) d x\right\| \leq \frac{K}{1+q} t\left(c_{0}\right)^{1+q}\left\|y_{0}-x_{0}\right\|^{1+q} .
$$

Taking norm on both sides of (2.9) and using (2.10),(2.11) and (2.12), we get

$$
\begin{aligned}
\left\|G\left(x_{1}\right)\right\| & \leq \frac{1}{\beta}\left(\frac{c_{0}}{1+q}+\left(c_{0}+1\right) t\left(c_{0}\right)+\frac{c_{0} t\left(c_{0}\right)^{1+q}}{1+q}\right)\left\|y_{0}-x_{0}\right\|, \\
& \leq \frac{1}{\beta} s\left(c_{0}\right)\left\|y_{0}-x_{0}\right\| .
\end{aligned}
$$

Using (2.8) and (2.13), we get

$$
\left\|y_{1}-x_{1}\right\| \leq\left\|\Gamma_{1}\right\|\left\|G\left(x_{1}\right)\right\| \leq r\left(c_{0}\right)\left\|\Gamma_{0}\right\|\left\|G\left(x_{1}\right)\right\| \leq r\left(c_{0}\right) s\left(c_{0}\right)\left\|y_{0}-x_{0}\right\| .
$$

Now

$$
\begin{aligned}
\left\|z_{1}-y_{1}\right\| & \leq 5\left\|\Gamma_{1}\right\| G\left(y_{1}\right)\|\| \leq 5\left\|\Gamma_{1}\right\| \int_{0}^{1}\left\|G^{\prime}\left(x_{1}+\theta\left(y_{1}-x_{1}\right)\right)-G^{\prime}\left(x_{1}\right)\right\|\left\|y_{1}-x_{1}\right\| d \theta, \\
& \leq 5 r\left(c_{0}\right)\left\|\Gamma_{0}\right\| \frac{K}{1+q}\left\|y_{1}-x_{1}\right\|^{1+q} \\
& \leq \frac{5 K \beta}{1+q} r\left(c_{0}\right)\left(r\left(c_{0}\right)\right)^{q}\left(s\left(c_{0}\right)\right)^{q}\left\|y_{0}-x_{0}\right\|^{q}\left\|y_{1}-x_{1}\right\|, \\
& \leq \frac{5}{1+q} c_{0}\left(r\left(c_{0}\right)\right)^{1+q}\left(s\left(c_{0}\right)\right)^{q}\left\|y_{1}-x_{1}\right\| .
\end{aligned}
$$

Therefore

$$
\left\|z_{1}-y_{1}\right\| \leq \frac{5}{1+q} c_{1}\left\|y_{1}-x_{1}\right\|
$$


Using (2.8) and (2.14), we get

$$
\begin{aligned}
K\left\|\Gamma_{1}\right\|\left\|y_{1}-x_{1}\right\|^{q} & \left.\leq K r\left(c_{0}\right)\left\|\Gamma_{0}\right\| r\left(c_{0}\right)\right)^{q}\left(s\left(c_{0}\right)\right)^{q}\left\|y_{0}-x_{0}\right\|^{q} \\
& \left.\leq K \beta \eta^{q} r\left(c_{0}\right)\right)^{1+q}\left(s\left(c_{0}\right)\right)^{q} \\
& \left.\leq c_{0} r\left(c_{0}\right)\right)^{1+q}\left(s\left(c_{0}\right)\right)^{q}=c_{1} .
\end{aligned}
$$

Therefore,

$$
K\left\|\Gamma_{1}\right\|\left\|y_{1}-x_{1}\right\|^{q} \leq c_{1} .
$$

For $n=1$, the recurrence relations (I)-(IV) follows from Eqs. (2.8), (2.14), (2.15) and (2.16) respectively. The recurrence relation $(\mathrm{V})$ is already proved in (2.7) for $n=1$. Assuming that (I)-(V) holds for $n=k$ and $x_{k}, y_{k}, z_{k} \in \Omega$, following in a similar manner given above, it can easily be proved that (I)-(V) holds for $n=k+1$. Hence recurrence relations hold for $n \geq 1$.

\subsection{Convergence Theorem}

In this subsection, a convergence theorem for $\left\{x_{n}\right\}$ is derived from (1.2) for solving (1.1). This will require use of Lemma 1, Lemma 2 and the recurrence relations established in the earlier subsection under the assumption given in (1),(2) and (3).

Theorem 2.1. Let $\Gamma_{0}=G^{\prime}\left(x_{0}\right)^{-1} \in \mathcal{L}(T, S)$ exists at $x_{0} \in D$ and the inequalities (1),(2) and (3) hold. Suppose $c_{0}=K \beta \eta^{q}<\tau_{1}$ and $B\left(x_{0}, R \eta\right)=\left\{x \in X:\left\|x-x_{0}\right\|<R \eta\right\} \subset D$, where $R=\frac{5}{1+q} c_{0}+\frac{\left(1+t\left(c_{0}\right)\right)^{q}}{1-r\left(c_{0}\right) s\left(c_{0}\right)}$. Now, starting with $x_{0},\left\{x_{n}\right\}$ derived by (1.2) converges to the solution $x^{*}$. In that case, $x_{n}, y_{n}, z_{n}$ lies in $\bar{B}\left(x_{0}, R \eta\right)$, and $x^{*}$ is the only solution of (1.1) in $B\left(x_{0}, r\right) \cap D$ where, $r=\left(\frac{1+q}{c_{0}}-R^{q}\right)^{\frac{1}{q}} \eta$.

Proof. To prove the Theorem 2.1, first, we show that $y_{n}$ and $z_{n} \in B\left(x_{0}, R \eta\right) \subset D$. Clearly, $\Gamma_{n}$ exists for all $n \geq 1$, since, $c_{0}<\tau_{1}$. Using recurrence relation $(\mathrm{V})$, we get

$$
\begin{aligned}
\left\|x_{n}-x_{0}\right\| & \leq\left\|x_{n}-x_{n-1}\right\|+\left\|x_{n-1}-x_{n-2}\right\|+\left\|x_{n-2}-x_{n-3}\right\|+\ldots+\left\|x_{1}-x_{0}\right\|, \\
& \leq\left(1+t\left(c_{n-1}\right)\right)^{q}\left\|y_{n-1}-x_{n-1}\right\|+\left(1+t\left(c_{n-2}\right)\right)^{q}\left\|y_{n-2}-x_{n-2}\right\| \\
& +\left(1+t\left(c_{n-3}\right)\right)^{q}\left\|y_{n-3}-x_{n-3}\right\|+\cdots+\left(1+t\left(c_{0}\right)\right)^{q}\left\|y_{0}-x_{0}\right\| .
\end{aligned}
$$

Since, $t(x)$ is an increasing function and $\left\{c_{n}\right\}$ is a decreasing sequence, this gives,

$$
\left\|x_{n}-x_{0}\right\| \leq\left(1+t\left(c_{0}\right)\right)^{q} \sum_{k=0}^{n-1}\left(r\left(c_{0}\right) s\left(c_{0}\right)\right)^{k}\left\|y_{0}-x_{0}\right\| .
$$


Now, using recurrence relation (II) and (2.18), we get

$$
\begin{aligned}
\left\|y_{n}-x_{0}\right\| & \leq\left\|y_{n}-x_{n}\right\|+\left\|x_{n}-x_{0}\right\| \\
& \leq\left(r\left(c_{n-1}\right) s\left(c_{n-1}\right)\right)\left\|y_{n-1}-x_{n-1}\right\|+\left(1+t\left(c_{0}\right)\right)^{q} \sum_{k=0}^{n-1}\left(r\left(c_{0}\right) s\left(c_{0}\right)\right)^{k}\left\|y_{0}-x_{0}\right\| \\
& \leq\left(r\left(c_{0}\right) s\left(c_{0}\right)\right)^{n}\left\|y_{0}-x_{0}\right\|+\left(1+t\left(c_{0}\right)\right)^{q} \sum_{k=0}^{n-1}\left(r\left(c_{0}\right) s\left(c_{0}\right)\right)^{k}\left\|y_{0}-x_{0}\right\|, \\
& \leq\left(1+t\left(c_{0}\right)\right)^{q}\left(r\left(c_{0}\right) s\left(c_{0}\right)\right)^{n}\left\|y_{0}-x_{0}\right\|+\left(1+t\left(c_{0}\right)\right)^{q} \sum_{k=0}^{n-1}\left(r\left(c_{0}\right) s\left(c_{0}\right)\right)^{k}\left\|y_{0}-x_{0}\right\|, \\
& \leq\left(1+t\left(c_{0}\right)\right)^{q} \sum_{k=0}^{n}\left(r\left(c_{0}\right) s\left(c_{0}\right)\right)^{k}\left\|y_{0}-x_{0}\right\|, \\
& \leq\left(1+t\left(c_{0}\right)\right)^{q} \frac{1-\left(r\left(c_{0}\right) s\left(c_{0}\right)\right)^{n+1}}{1-r\left(c_{0}\right) s\left(c_{0}\right)}\left\|y_{0}-x_{0}\right\|<R \eta .
\end{aligned}
$$

Using recurrence relation (III) and (2.19), we get

$$
\begin{aligned}
\left\|z_{n}-x_{0}\right\| & \leq\left\|z_{n}-y_{n}\right\|+\left\|y_{n}-x_{0}\right\| \\
& \leq \frac{5}{1+q} c_{0}\left(r\left(c_{0}\right) s\left(c_{0}\right)\right)^{n}\left\|y_{0}-x_{0}\right\|+\left(1+t\left(c_{0}\right)\right)^{q} \frac{1-\left(r\left(c_{0}\right) s\left(c_{0}\right)\right)^{n+1}}{1-r\left(c_{0}\right) s\left(c_{0}\right)}\left\|y_{0}-x_{0}\right\| \\
& \leq\left(\frac{5}{1+q} c_{0}+\left(1+t\left(c_{0}\right)\right)^{q} \frac{1-\left(r\left(c_{0}\right) s\left(c_{0}\right)\right)^{n+1}}{1-r\left(c_{0}\right) s\left(c_{0}\right)}\right)\left\|y_{0}-x_{0}\right\| . \\
& \leq\left(\frac{5}{1+q} c_{0}+\left(1+t\left(c_{0}\right)\right)^{q} \frac{1-\left(r\left(c_{0}\right) s\left(c_{0}\right)\right)^{n+1}}{1-r\left(c_{0}\right) s\left(c_{0}\right)}\right) \eta<R \eta .
\end{aligned}
$$

Hence $y_{n}$ and $z_{n} \in \Omega$. Now

$$
\begin{aligned}
\left\|x_{n+1}-x_{n}\right\| & \leq\left(1+t\left(c_{n}\right)\right)^{q}\left\|y_{n}-x_{n}\right\| \\
& \leq\left(1+t\left(c_{n}\right)\right)^{q} r\left(c_{n-1}\right) s\left(c_{n-1}\right)\left\|y_{n-1}-x_{n-1}\right\| \\
& \leq \cdots \leq\left(1+t\left(c_{n}\right)\right)^{q}\left(\prod_{j=0}^{n-1} r\left(c_{j}\right) s\left(c_{j}\right)\right)\left\|y_{0}-x_{0}\right\|
\end{aligned}
$$

For the convergence of $\left\{x_{n}\right\}$, we have to show that $\left\{x_{n}\right\}$ is a cauchy sequence. For this, consider

$$
\begin{aligned}
\left\|x_{n+m}-x_{n}\right\| & \leq\left\|x_{n+m}-x_{n+m-1}\right\|+\left\|x_{n+m-1}-x_{n+m-2}\right\|+\left\|x_{n+m-2}-x_{n+m-3}\right\|+\ldots+\left\|x_{n+1}-x_{n}\right\| \\
& \leq\left(1+t\left(c_{n+m-1}\right)\right)^{q}\left(\prod_{j=0}^{n+m-2} r\left(c_{j}\right) s\left(c_{j}\right)\right) \eta+\left(1+t\left(c_{n+m-2}\right)\right)^{q}\left(\prod_{j=0}^{n+m-3} r\left(c_{j}\right) s\left(c_{j}\right)\right) \eta \\
& +\left(1+t\left(c_{n+m-3}\right)\right)^{q}\left(\prod_{j=0}^{n+m-4} r\left(c_{j}\right) s\left(c_{j}\right)\right) \eta+\ldots+\left(1+t\left(c_{n}\right)\right)^{q}\left(\prod_{j=0}^{n-1} r\left(c_{j}\right) s\left(c_{j}\right)\right) \eta
\end{aligned}
$$

This gives

$$
\begin{aligned}
\left\|x_{n+m}-x_{n}\right\| & \leq\left(1+t\left(c_{0}\right)\right)^{q} \sum_{l=0}^{m-1}\left(\prod_{j=0}^{n+l-1} r\left(c_{j}\right) s\left(c_{j}\right)\right) \eta \\
& \leq\left(1+t\left(c_{0}\right)\right)^{q} \sum_{l=0}^{m-1}\left(r\left(c_{0}\right) s\left(c_{0}\right)\right)^{l+n} \eta
\end{aligned}
$$


Therefore

$$
\left\|x_{n+m}-x_{n}\right\| \leq\left(1+t\left(c_{0}\right)\right)^{q} \frac{1-\left(r\left(c_{0}\right) s\left(c_{0}\right)\right)^{m}}{1-r\left(c_{0}\right) s\left(c_{0}\right)}\left(r\left(c_{0}\right) s\left(c_{0}\right)\right)^{n} \eta .
$$

Hence $\left\{x_{n}\right\}$ is a cauchy sequence if $r\left(c_{0}\right) s\left(c_{0}\right)<1$ and hence convergent.

For $n=0$ and $m \geq 1$, we get

$$
\left\|x_{m}-x_{0}\right\| \leq\left(1+t\left(c_{0}\right)\right)^{q} \frac{1-\left(r\left(c_{0}\right) s\left(c_{0}\right)\right)^{m}}{1-r\left(c_{0}\right) s\left(c_{0}\right)}<R \eta .
$$

Hence $x_{m} \in \bar{B}\left(x_{0}, R \eta\right)$. Taking $m \rightarrow \infty$ in $(2.20)$, we get $x^{*} \in \bar{B}\left(x_{0}, R \eta\right)$. Now, it is to be shown that $x^{*}$ is a solution of (1.1). From (1.2), we have $y_{n}=x_{n}-\Gamma_{n} G\left(x_{n}\right)$. This gives

$$
\begin{aligned}
\left\|G\left(x_{n}\right)\right\| & \leq\left\|G^{\prime}\left(x_{n}\right)\right\|\left\|y_{n}-x_{n}\right\| \\
& \leq\left\|G^{\prime}\left(x_{n}\right)\right\| r\left(c_{n-1}\right) s\left(c_{n-1}\right)\left\|y_{n-1}-x_{n-1}\right\| \\
& \leq\left\|G^{\prime}\left(x_{n}\right)\right\|\left(r\left(c_{0}\right) s\left(c_{0}\right)\right)^{n}\left\|y_{0}-x_{0}\right\| .
\end{aligned}
$$

Now,

$$
\begin{aligned}
\left\|G^{\prime}\left(x_{n}\right)\right\| & \leq\left\|G^{\prime}\left(x_{n}\right)-G^{\prime}\left(x_{0}\right)\right\|+\left\|G^{\prime}\left(x_{0}\right)\right\| \\
& \leq K\left\|x_{n}-x_{0}\right\|^{q}+\left\|G^{\prime}\left(x_{0}\right)\right\| \\
& <K(R \eta)^{q}+\left\|G^{\prime}\left(x_{0}\right)\right\|
\end{aligned}
$$

Therefore, $\left\|G^{\prime}\left(x_{n}\right)\right\|$ is bounded. Hence from (2.21), we obtain that $\left\|G\left(x_{n}\right)\right\| \rightarrow 0$ as $n \rightarrow \infty$. Thus by the continuity of $G$ in $D$, we get $G\left(x^{*}\right)=0$. For the uniqueness, let there exists $z^{*} \in B\left(x_{0}, r\right)$ such that $G\left(z^{*}\right)=0, z^{*} \neq x^{*}$. Then

$$
0=G\left(z^{*}\right)-G\left(x^{*}\right)=\int_{0}^{1} G^{\prime}\left(x^{*}+\theta\left(z^{*}-x^{*}\right)\right) d \theta\left(z^{*}-x^{*}\right)=P\left(z^{*}-x^{*}\right)
$$

Now, we have to show that the inverse of $P=\int_{0}^{1} G^{\prime}\left(x^{*}+\theta\left(z^{*}-x^{*}\right)\right) d \theta$ exists and $z^{*}=x^{*}$. Consider

$$
\begin{aligned}
\left\|I-\Gamma_{0} P\right\| & \leq\left\|\Gamma_{0}\right\| \int_{0}^{1}\left\|\left(G^{\prime}\left(x^{*}+\theta\left(z^{*}-x^{*}\right)\right)-G^{\prime}\left(x_{0}\right)\right)\right\| d \theta \\
& \leq K \beta \int_{0}^{1}\left\|x^{*}+\theta\left(z^{*}-x^{*}\right)-x_{0}\right\|^{q} d \theta \\
& \leq K \beta \int_{0}^{1}\left\|(1-\theta)\left(x^{*}-x_{0}\right)+\theta\left(z^{*}-x_{0}\right)\right\|^{q} d \theta \\
& \leq \frac{K \beta}{1+q}\left(\left\|x^{*}-x_{0}\right\|^{q}+\left\|z^{*}-x_{0}\right\|^{q}\right) \\
& \leq \frac{K \beta}{1+q}\left((R \eta)^{q}+r^{q}\right) \\
& =1
\end{aligned}
$$

Thus, $\left\|I-\Gamma_{0} P\right\|<1$. Therefore, $P^{-1}$ exists and hence $z^{*}=x^{*}$.

\subsection{Numerical examples}

In this subsection, a number of numerical examples are solved to demonstrate the efficacy of our semilocal convergence analysis. 
Example 2.1. Consider $G(x)=0$, where

$$
G(x)(s)=x(s)-1-\frac{1}{3} \int_{0}^{1} G_{1}(s, u)(x(u))^{1+q} d u,
$$

$s \in[0,1], q \in(0,1]$ and $x \in C[0,1]$ be an integral equation. The continuous and nonnegative kernel $G_{1}$ in $[0,1] \times[0,1]$ is given by

$$
G_{1}(s, u)= \begin{cases}(1-s) u, & t \leq u, \\ s(1-u), & s \leq u .\end{cases}
$$

Using sup-norm, we get

$$
\begin{aligned}
\left\|G^{\prime}(x)-G^{\prime}(y)\right\| & \leq \frac{1+q}{3} \max _{s \in[0,1]}\left|\int_{0}^{1} G_{1}(s, u) d u\right|\left\|x(u)^{q}-y(u)^{q}\right\| \\
& \leq \frac{1+q}{24}\|x-y\|^{q}
\end{aligned}
$$

Thus, the Lipschitz condition for $G^{\prime}$ fails for $q \in(0,1)$. Now, $\left\|G\left(x_{0}\right)\right\| \leq\left\|x_{0}-1\right\|+\frac{1}{24}\left\|x_{0}\right\|^{1+q}$ and $\left\|I-G^{\prime}\left(x_{0}\right)\right\| \leq \frac{1+q}{24}\left\|x_{0}\right\|^{q}$. If $\frac{1+q}{24}\left\|x_{0}\right\|^{q}<1$ then by Banach Lemma, we obtain

$$
\left\|\Gamma_{0}\right\|=\left\|G^{\prime}\left(x_{0}\right)^{-1}\right\| \leq \frac{1}{1-\frac{1+q}{24}\left\|x_{0}\right\|^{q}}=\beta .
$$

Hence,

$$
\left\|\Gamma_{0} G\left(x_{0}\right)\right\| \leq \frac{\left\|x_{0}-1\right\|+\frac{1}{24}\left\|x_{0}\right\|^{1+q}}{1-\frac{1+q}{16}\left\|x_{0}\right\|^{q}}=\eta .
$$

Now for $q=0.85$ and $x_{0}=x_{0}(s)=1$ in $[0,1]$, we get $c_{0}=K \beta \eta^{q}=0.00600<\tau_{1}=0.27523$. Therefore, conditions of Lemma 1, Lemma 2 and Theorem 1 are satisfied. Hence the existence of $x^{*}$ is guaranteed in $\bar{B}\left(x_{0}, 0.04630\right)$ and the uniqueness in $B\left(x_{0}, 38.25348\right)$. It is to be noted that if we consider $q=1$ in (2.22), we get

$$
G(x)(s)=x(s)-1-\frac{1}{3} \int_{0}^{1} G_{1}(s, u)(x(u))^{2} d u
$$

Therefore,

$$
\left\|G^{\prime}(x)-G^{\prime}(y)\right\| \leq \frac{1}{12}\|x-y\|
$$

Again, starting with $x_{0}=x_{0}(s)=1$ in $[0,1]$, we get $a_{0}=0.00413<\tau_{1}=0.29314$. Therefore, conditions of Lemma 1, Lemma 2 and Theorem 1 are satisfied. Hence the existence of $x^{*}$ is guaranteed in $\bar{B}\left(x_{0}, 0.04620\right)$ and the uniqueness in $B\left(x_{0}, 21.99790\right)$. Thus, improved existence and uniqueness domains of convergence balls are obtained under Hölder condition in comparison to the Lipschitz condition.

Example 2.2. Consider $G(x)=0$, where

$$
G(x)(s)=x(s)-1-\frac{1}{4} \int_{0}^{1} \frac{s}{s+u}(x(u))^{1+q} d u,
$$

$s \in[0,1], q \in(0,1]$ and $x \in C[0,1]$ be the integral equation. 
Using sup-norm, we get

$$
\begin{aligned}
\left\|G^{\prime}(x)-G^{\prime}(y)\right\| & \leq \frac{1+q}{4} \max _{s \in[0,1]}\left|\int_{0}^{1} \frac{s}{s+u} d u\right|\left\|x(u)^{q}-y(u)^{q}\right\| \\
& \leq \frac{1+q}{4} \log 2\|x-y\|^{q}
\end{aligned}
$$

Thus, the Lipschitz condition for $G^{\prime}$ fails for $q \in(0,1)$. Now, $\left\|G\left(x_{0}\right)\right\| \leq\left\|x_{0}-1\right\|+\frac{\log 2}{4}\left\|x_{0}\right\|^{1+q}$ and $\left\|I-G^{\prime}\left(x_{0}\right)\right\| \leq \frac{1+q}{4} \log 2\left\|x_{0}\right\|^{q}$. If $\frac{1+q}{4} \log 2\left\|x_{0}\right\|^{q}<1$ then by Banach Lemma, we obtain

$$
\left\|\Gamma_{0}\right\|=\left\|G^{\prime}\left(x_{0}\right)^{-1}\right\| \leq \frac{1}{1-\frac{1+q}{4} \log 2\left\|x_{0}\right\|^{q}}=\beta
$$

Hence,

$$
\left\|\Gamma_{0} G\left(x_{0}\right)\right\| \leq \frac{\left\|x_{0}-1\right\|+\log 2\left\|x_{0}\right\|^{1+q}}{1-\frac{1+q}{4} \log 2\left\|x_{0}\right\|^{q}}=\eta .
$$

Now, for $q=0.7$ and $x_{0}=x_{0}(s)=1$ in $[0,1]$, we get $c_{0}=K \beta \eta^{q}=0.15631<\tau_{1}=0.25510$. Therefore, conditions of Lemma 1, Lemma 2 and Theorem 1 are satisfied. Hence the existence of $x^{*}$ is guaranteed in $\bar{B}\left(x_{0}, 0.47873\right)$ and the uniqueness in $B\left(x_{0}, 6.85383\right)$. It is to be noted that if we consider $q=1$ in (2.23), we get

$$
G(x)(s)=x(s)-1-\frac{1}{4} \int_{0}^{1} \frac{s}{s+u}(x(u))^{2} d u
$$

Therefore,

$$
\left\|G^{\prime}(x)-G^{\prime}(y)\right\| \leq \frac{1}{2} \log 2\|x-y\|
$$

Again, starting with $x_{0}=x_{0}(s)=1$ in $[0,1]$, we get $c_{0}=0.14066<\tau_{1}=0.29314$. Therefore, conditions of Lemma 1, Lemma 2 and Theorem 1 are satisfied. Hence the existence of $x^{*}$ is guaranteed in $\bar{B}\left(x_{0}, 0.44916\right)$ and the uniqueness in $B\left(x_{0}, 3.65166\right)$. Here also, improved existence and uniqueness domains of convergence balls are obtained under Hölder condition in comparison to the Lipschitz condition.

Example 2.3. Consider the following nonlinear BVP

$$
x^{\prime \prime}+x^{1+q}=0, \quad q \in(0,1], \quad x(0)=x(1)=0 .
$$

The interval $[0,1]$ is divided into $N$ subintervals with points $t_{i}=i h, i=0,1, \ldots, N$ where $h=\frac{1}{N}$. Approximating the second derivative by central difference scheme given by $x_{i}^{\prime \prime} \approx \frac{x_{i-1}-2 x_{i}+x_{i+1}}{h^{2}}, \quad i=$ $1,2, \ldots, N-1$ in $(2.24)$, we get

$$
-x_{i-1}+2 x_{i}-x_{i+1}-h^{2} x_{i}^{1+q}=0 .
$$

This can be expressed as $G(x)=H x-h^{2} l(x)=0$, where, $G: \mathbb{R}^{N-1} \rightarrow \mathbb{R}^{N-1}, x=\left(x_{1}, x_{2}, \ldots, x_{N-1}\right)^{t}$, $l(x)=\left(x_{1}^{1+q}, x_{2}^{1+q}, \ldots, x_{N-1}^{1+q}\right)^{t}$ and the matrix $H$ is given by

$$
H=\left[\begin{array}{ccccc}
2 & -1 & 0 & \ldots & 0 \\
-1 & 2 & -1 & \ldots & 0 \\
0 & -1 & 2 & \ldots & 0 \\
\vdots & \vdots & \vdots & \ddots & 0 \\
0 & 0 & 0 & \ldots & 2
\end{array}\right]
$$


Here $G^{\prime}(x)=H-(1+q) h^{2} M(x)$ where, $M(x)=\operatorname{diag}\left\{x_{1}^{q}, x_{2}^{q}, \ldots, x_{N-1}^{q}\right\}$ and $\left\|G^{\prime}(x)-G^{\prime}(y)\right\| \leq$ $(1+q) h^{2}\|x-y\|^{q}$. Choosing $h=\frac{1}{10}, q=\frac{1}{2}$ and

$$
x_{0}=(33.5739,65.2025,91.566,109.168,115.363,109.168,91.566,65.2025,33.5739)^{t},
$$

we get $K=0.015, \beta=26.5888$ and $\eta=3.7570 \times 10^{-4}$. Hence $c_{0}=K \beta \eta^{\frac{1}{2}}=0.0077<\tau_{1}=0.2234$. Therefore, conditions of Lemma 1, Lemma 2 and Theorem 1 are satisfied. Hence the existence of $x^{*}$ is guaranteed in $\bar{B}\left(x_{0}, 0.00039\right)$ and the uniqueness in $B\left(x_{0}, 13.99678\right)$. The domains of existence and unique balls obtained in [17] are $\bar{B}\left(x_{0}, 0.0000247488\right)$ and $B\left(x_{0}, 3.51756\right)$ respectively which are inferior to those obtained by us under similar conditions.

\section{Local convergence analysis}

In this Section, the local convergence analysis of a fifth order iteration (1.2) for (1.1) in the Banach space setting under Hölder condition on $G^{\prime}$ is studied. Let $K_{0}>0, K_{1}>0, q \in(0,1]$ and the following assumptions hold when $x, y \in D$.

$$
\begin{aligned}
G\left(x^{*}\right)=0, \quad G^{\prime}\left(x^{*}\right)^{-1} & \in \mathcal{L}(T, S) \\
\left\|G^{\prime}\left(x^{*}\right)^{-1}\left(G^{\prime}(x)-G^{\prime}\left(x^{*}\right)\right)\right\| & \leq K_{0}\left\|x-x^{*}\right\|^{q} \\
\left\|G^{\prime}\left(x^{*}\right)^{-1}\left(G^{\prime}(x)-G^{\prime}(y)\right)\right\| & \leq K_{1}\|x-y\|^{q} .
\end{aligned}
$$

The local convergence analysis in [11] uses an additional condition $\left\|G^{\prime}\left(x^{*}\right)^{-1} G^{\prime}(x)\right\| \leq M$ for $M>0$ and remarked that this can be dropped. This condition is not used in our work.

Lemma 3.1. If $G$ satisfies (3.1)-(3.2) then the inequalities given below hold for $x \in D_{0}$ and $\theta \in[0,1]$

$$
\begin{aligned}
\left\|G^{\prime}\left(x^{*}\right)^{-1} G^{\prime}(x)\right\| & \leq 1+K_{0}\left\|x-x^{*}\right\|^{q}, \\
\| G^{\prime}\left(x^{*}\right)^{-1}\left(G^{\prime}\left(x^{*}+\theta\left(x-x^{*}\right)\right) \|\right. & \leq 1+K_{0}\left\|x-x^{*}\right\|^{q}, \\
\left\|G^{\prime}\left(x^{*}\right)^{-1} G(x)\right\| & \leq\left(1+K_{0}\left\|x-x^{*}\right\|^{q}\right)\left\|x-x^{*}\right\| .
\end{aligned}
$$

Proof. The proof is trivial.

The following theorem describes the convergence analysis locally of (1.2).

Theorem 3.1. Suppose $x^{*} \in D_{0}$ such that (3.1) and (3.2) hold. Then, $\left\{x_{n}\right\}$ given by (1.2) is defined, remains in $B\left(x^{*}, \rho\right)$ for $x_{0} \in B\left(x^{*}, \rho\right)$ with appropriate $\rho$ and converges to $x^{*}$. Also, the estimates given below hold for all $n \geq 0$.

$$
\begin{aligned}
\left\|y_{n}-x^{*}\right\| & \leq \gamma_{1}\left(\left\|x_{n}-x^{*}\right\|\right)\left\|x_{n}-x^{*}\right\|<\rho, \\
\left\|z_{n}-x^{*}\right\| & \leq \gamma_{2}\left(\left\|x_{n}-x^{*}\right\|\right)\left\|x_{n}-x^{*}\right\|<\rho, \\
\left\|x_{n+1}-x^{*}\right\| & \leq \gamma_{3}\left(\left\|x_{n}-x^{*}\right\|\right)\left\|x_{n}-x^{*}\right\|<\rho,
\end{aligned}
$$

where $\gamma_{1}, \gamma_{2}$ and $\gamma_{3}$ are the functions to be defined. Furthermore, if $\exists R \in\left[\rho, \frac{1+q}{K_{0}}\right)$ such that $\bar{B}\left(x^{*}, R\right) \subseteq$ $D_{0}$, then $x^{*}$ is unique in $\bar{B}\left(x^{*}, R\right)$.

Proof. Using $x_{0} \in \Omega_{0}$ and (3.1), we get

$$
\left\|I-G^{\prime}\left(x^{*}\right)^{-1} G^{\prime}\left(x_{0}\right)\right\| \leq\left\|G^{\prime}\left(x^{*}\right)^{-1}\left(G^{\prime}\left(x_{0}\right)-G^{\prime}\left(x^{*}\right)\right)\right\| \leq K_{0}\left\|x_{0}-x^{*}\right\|^{q}<1
$$


for $\left\|x_{0}-x^{*}\right\|^{q}<\frac{1}{K_{0}}$. Therefore, by Banach Lemma, $\Gamma_{0}$ exists and

$$
\left\|\Gamma_{0} G^{\prime}\left(x^{*}\right)\right\| \leq \frac{1}{1-K_{0}\left\|x_{0}-x^{*}\right\|^{q}} .
$$

Therefore, $y_{0}$ is well defined and hence $z_{0}$ and $x_{1}$ are well defined.

From (1.2) for $n=0$, this gives

$$
\begin{aligned}
y_{0}-x^{*} & =-\Gamma_{0}\left(G\left(x_{0}\right)-G^{\prime}\left(x_{0}\right)\left(x_{0}-x^{*}\right)\right) \\
& =-\Gamma_{0} G^{\prime}\left(x^{*}\right) \int_{0}^{1} G^{\prime}\left(x^{*}\right)^{-1}\left[G^{\prime}\left(x^{*}+\theta\left(x_{0}-x^{*}\right)\right)-G^{\prime}\left(x_{0}\right)\right]\left(x_{0}-x^{*}\right) d \theta
\end{aligned}
$$

Taking norm on both sides and using (3.2) and (3.9), we get

$$
\begin{aligned}
\left\|y_{0}-x^{*}\right\| & \leq \frac{K_{1}\left\|x_{0}-x^{*}\right\|^{q}}{\left(1-K_{0}\left\|x_{0}-x^{*}\right\|^{q}\right)(1+q)}\left\|x_{0}-x^{*}\right\| \\
& \leq \gamma_{1}\left(\left\|x_{0}-x^{*}\right\|\right)\left\|x_{0}-x^{*}\right\|
\end{aligned}
$$

where,

$$
\gamma_{1}(\theta)=\frac{K_{1} \theta^{q}}{\left(1-K_{0} \theta^{q}\right)(1+q)} .
$$

Consider $\eta_{1}(\theta)=\gamma_{1}(\theta)-1$. Since $\eta_{1}(0)=-1<0$ and $\eta_{1}\left(\left(\frac{1}{K_{0}}\right)^{1 / q}\right) \rightarrow+\infty$. Therefore, by intermediate value theorem, $\eta_{1}(\theta)$ has at least one root in $\left(0,\left(\frac{1}{K_{0}}\right)^{1 / q}\right)$. Let $r_{1}$ be the smallest root of $\eta_{1}(\theta)$ in this interval. Then, we get $0<r_{1}<\left(\frac{1}{K_{0}}\right)^{1 / q}$, and $0 \leq \gamma_{1}(\theta)<1, \forall \quad \theta \in\left[0, r_{1}\right)$. Thus, we have

$$
\left\|y_{0}-x^{*}\right\| \leq \gamma_{1}\left(\left\|x_{0}-x^{*}\right\|\right)\left\|x_{0}-x^{*}\right\|<\left\|x_{0}-x^{*}\right\| .
$$

Again, from (1.2) for $n=0$, and by using (3.5) and (3.9), we get

$$
\begin{aligned}
\left\|z_{0}-x^{*}\right\| & \leq\left\|y_{0}-x^{*}\right\|+5\left\|\Gamma_{0} G\left(y_{0}\right)\right\| \\
& \leq\left\|y_{0}-x^{*}\right\|+5\left\|\Gamma_{0} G^{\prime}\left(x^{*}\right)\right\|\left\|G^{\prime}\left(x^{*}\right)^{-1} G\left(y_{0}\right)\right\|, \\
& \leq\left[1+5\left(\frac{1+K_{0}\left\|y_{0}-x^{*}\right\|^{q}}{1-K_{0}\left\|x_{0}-x^{*}\right\|^{q}}\right)\right]\left\|y_{0}-x^{*}\right\| \\
& \leq\left[1+5\left(\frac{\left.1+K_{0}\left(\gamma_{1}\left(\left\|x_{0}-x^{*}\right\|\right)\right)^{q}\left\|x_{0}-x^{*}\right\|^{q}\right)}{1-K_{0}\left\|x_{0}-x^{*}\right\|^{q}}\right)\right] \gamma_{1}\left(\left\|x_{0}-x^{*}\right\|\right)\left\|x_{0}-x^{*}\right\| \\
& \leq \gamma_{2}\left(\left\|x_{0}-x^{*}\right\|\right)\left\|x_{0}-x^{*}\right\|,
\end{aligned}
$$

where,

$$
\gamma_{2}(\theta)=\left[1+5\left(\frac{1+K_{0}\left(\gamma_{1}(\theta)\right)^{q} \theta^{q}}{1-K_{0} \theta^{q}}\right)\right] \gamma_{1}(\theta)
$$

Consider $\eta_{2}(\theta)=\gamma_{2}(\theta)-1$. Then $\eta_{2}(0)=-1<0$ and $\eta_{2}\left(r_{1}\right)=5\left(\frac{1+K_{0} r_{1}^{q}}{1-K_{0} r_{1}^{q}}\right)>0$. Therefore, $\eta_{2}(\theta)$ has at least one root in $\left(0, r_{1}\right)$. Let $r_{2}$ be the smallest root of $\eta_{2}(\theta)$ in this interval. Therefore, $0<r_{2}<r_{1}$ and $0 \leq \gamma_{2}(\theta)<1, \forall \theta \in\left[0, r_{2}\right)$. Thus, we get

$$
\left\|z_{0}-x^{*}\right\| \leq \gamma_{2}\left(\left\|x_{0}-x^{*}\right\|\right)\left\|x_{0}-x^{*}\right\|<\left\|x_{0}-x^{*}\right\| .
$$


Again, from (1.2) for $n=0$, and by using (3.5) and (3.9), we get

$$
\begin{aligned}
\left\|x_{1}-x^{*}\right\| \leq & \left\|z_{0}-x^{*}\right\|+\frac{1}{5}\left\|\Gamma_{0}\left(-16 G\left(y_{0}\right)+G\left(z_{0}\right)\right)\right\| \\
\leq & \left\|z_{0}-x^{*}\right\|+\frac{1}{5}\left\|\Gamma_{0} G^{\prime}\left(x^{*}\right)\right\|\left(16\left\|G^{\prime}\left(x^{*}\right)^{-1} G\left(y_{0}\right)\right\|+\left\|G^{\prime}\left(x^{*}\right)^{-1} G\left(z_{0}\right)\right\|\right), \\
\leq & {\left[\gamma_{2}\left(\left\|x_{0}-x^{*}\right\|\right)\left\|x_{0}-x^{*}\right\|+\frac{1}{5} \frac{1}{1-K_{0}\left\|x_{0}-x^{*}\right\|^{q}}\left(16\left(1+K_{0}\left(\gamma_{1}\left(\left\|x_{0}-x^{*}\right\|\right)\right)^{q}\left\|x_{0}-x^{*}\right\|^{q}\right)\right.\right.} \\
& \left.\left.\gamma_{1}\left(\left\|x_{0}-x^{*}\right\|\right)\left\|x_{0}-x^{*}\right\|+\left(1+K_{0}\left(\gamma_{2}\left(\left\|x_{0}-x^{*}\right\|\right)\right)^{q}\left\|x_{0}-x^{*}\right\|^{q}\right) \gamma_{2}\left(\left\|x_{0}-x^{*}\right\|\right)\left\|x_{0}-x^{*}\right\|\right)\right] \\
\leq & \gamma_{3}\left(\left\|x_{0}-x^{*}\right\|\right)\left\|x_{0}-x^{*}\right\|,
\end{aligned}
$$

where,

$$
\gamma_{3}(\theta)=\left[\gamma_{2}(\theta)+\frac{1}{5\left(1-K_{0} \theta^{q}\right)}\left(16\left(1+K_{0}\left(\gamma_{1}(\theta)\right)^{q} \theta^{q}\right) \gamma_{1}(\theta)+\left(1+K_{0}\left(\gamma_{2}(\theta)\right)^{q} \theta^{q}\right) \gamma_{2}(\theta)\right)\right] .
$$

Consider $\eta_{3}(\theta)=\gamma_{3}(\theta)-1$. Since $\eta_{3}(0)=-1<0$ and

$$
\eta_{3}\left(r_{2}\right)=\frac{1}{5\left(1-K_{0} r_{2}^{q}\right)}\left(16\left(1+K_{0}\left(\gamma_{1}\left(r_{2}\right)\right)^{q} r_{2}^{q}\right) \gamma_{1}\left(r_{2}\right)+\left(1+K_{0}\left(\gamma_{2}\left(r_{2}\right)\right)^{q} r_{2}^{q}\right)\right)>0 .
$$

Therefore, $\eta_{3}(\theta)$ has atleast one root in $\left(0, r_{2}\right)$. Let $\rho$ be the smallest root of $\eta_{3}(\theta)$ in this interval and we get $0<\rho<r_{2}<r_{1}<\left(\frac{1}{K_{0}}\right)^{1 / q}$ and $0 \leq \gamma_{3}(\theta)<1, \forall \theta \in[0, \rho)$. Thus, we get

$$
\left\|x_{1}-x^{*}\right\| \leq \gamma_{3}\left(\left\|x_{0}-x^{*}\right\|\right)\left\|x_{0}-x^{*}\right\|<\left\|x_{0}-x^{*}\right\|<\rho .
$$

Therefore, theorem holds for $n=0$. Changing $x_{0}, y_{0}, z_{0}$ and $x_{1}$ by $x_{n}, y_{n}, z_{n}, x_{n+1}$ in the preceding way, we get the inequalities (3.6)-(3.8). Using the estimate $\left\|x_{n+1}-x^{*}\right\|<\left\|x_{n}-x^{*}\right\|<\rho$, we get $x_{n+1} \in B\left(x^{*}, \rho\right)$. Obviously the function $\gamma_{3}$ is increasing in its domain, so we have

$$
\begin{aligned}
\left\|x_{n+1}-x^{*}\right\| & \leq \gamma_{3}(\theta)\left\|x_{n}-x^{*}\right\| \leq \gamma_{3}(\theta) \gamma_{3}\left(\left\|x_{n-1}-x^{*}\right\|\right)\left\|x_{n-1}-x^{*}\right\| \\
& \leq \gamma_{3}(\theta)^{2} \gamma_{3}\left(\left\|x_{n-2}-x^{*}\right\|\right)\left\|x_{n-2}-x^{*}\right\| \leq \ldots \leq \gamma_{3}(\theta)^{n+1}\left\|x_{0}-x^{*}\right\| .
\end{aligned}
$$

Since $\gamma_{3}(\theta)<1 \forall \theta \in[0, \rho)$. Then $\lim _{n \rightarrow \infty} x_{n}=x^{*}$ as $\lim _{n \rightarrow \infty} \gamma_{3}(\theta)^{n+1}=0$. For the uniqueness, let $z^{*} \in B\left(x^{*}, \rho\right), z^{*} \neq x^{*}$ with $G\left(z^{*}\right)=0$.

Take the integral operator $Q=\int_{0}^{1} G^{\prime}\left(z^{*}+\theta\left(x^{*}-z^{*}\right)\right) d \theta$. Then by using (3.1), we have

$$
\left\|G^{\prime}\left(x^{*}\right)^{-1}\left(Q-G^{\prime}\left(x^{*}\right)\right)\right\| \leq \int_{0}^{1} K_{0}\left\|z^{*}+\theta\left(x^{*}-z^{*}\right)-x^{*}\right\|^{q} d \theta \leq \frac{K_{0}}{1+q}\left\|x^{*}-z^{*}\right\|^{q}=\frac{K_{0}}{1+q} R<1,
$$

therefore, by Banach Lemma $Q^{-1}$ exists. Then, from the identity

$$
0=G\left(x^{*}\right)-G\left(z^{*}\right)=Q\left(x^{*}-z^{*}\right)
$$

we obtain $x^{*}=z^{*}$.

\subsection{Numerical examples}

In this subsection, a number of examples are solved to demonstrate the efficacy of our approach. 
Example 3.1. Consider the function $G$ defined on $D_{0}=\left[-\frac{1}{2}, \frac{5}{2}\right]$ by

$$
G(x)= \begin{cases}x^{3} \ln x^{2}+x^{5}-x^{4}, & x \neq 0 \\ 0, & x=0\end{cases}
$$

The unique solution is $x^{*}=1$. The successive derivatives of $f$ are

$$
\begin{aligned}
G^{\prime}(x) & =3 x^{2} \ln x^{2}+5 x^{4}-4 x^{3}+2 x^{2}, \\
G^{\prime \prime}(x) & =6 x \ln x^{2}+20 x^{3}-12 x^{2}+10 x, \\
G^{\prime \prime \prime}(x) & =6 \ln x^{2}+60 x^{2}-24 x+22 .
\end{aligned}
$$

It can be easily observed that $G^{\prime \prime \prime}$ is unbounded on $D_{0}$. However, all the conditions of the iterative method (1.2) are satisfied and hence applying Theorem (3.1) with $x^{*}=1$, we obtained $K_{0}=K_{1}=$ 96.6628. Taking $q=0.4$, we get

$$
\rho=3.7 \times 10^{-8}<r_{2}=1.1 \times 10^{-7}<r_{1}=2.8 \times 10^{-6} .
$$

Example 3.2. Consider the function $G$ defined on $D_{0}=[1,3]$ by

$$
G(x)=\frac{2}{3} x^{\frac{3}{2}}-x
$$

For $x^{*}=\frac{9}{4}$, we obtained $K_{0}=K_{1}=1$. Taking $q=0.5$, we get

$$
\rho=0.012069<r_{2}=0.028479<r_{1}=0.36100 \text {. }
$$

Example 3.3. Consider the function $G$ defined on $D_{0}=\bar{B}(0,1)$ for $x=\left(x_{1}, x_{2}, x_{3}\right)^{t}$ by

$$
G(x)=\left(e^{x_{1}}-1, \frac{e-1}{2} x_{2}^{2}+x_{2}, x_{3}\right)^{t} .
$$

For $x^{*}=(0,0,0)$, we obtained $K_{0}=e-1$ and $K_{1}=e$. Taking $q=0.7$, we get

$$
\rho=0.013577<r_{2}=0.026019<r_{1}=0.180313 .
$$

Example 3.4. Consider the Hammerstein integral equation given by

$$
G(x(s))=x(s)-5 \int_{0}^{1} s u x(u)^{3} d u
$$

with $x(s)$ in $\mathcal{C}[0,1]$.

So, we obtained $K_{0}=7.5$ and $K_{1}=15$. Taking $q=0.5$, we get

$$
\rho=6.8 \times 10^{-5}<r_{2}=1.8 \times 10^{-4}<r_{1}=0.003265 .
$$

We have also compared the radius of convergence ball for $q=0.5$ obtained by method (1.2) with existing method (1.3) in Table 2. It can be easily observed that the present method gives large radius of convergence ball as compared to method (1.3). 
Table 1: Values of parameters

\begin{tabular}{|c|c|c|c|}
\hline Examples & $q$ & $\lambda$ & $\alpha$ \\
\hline 3.1 & 0.4 & 1 & -1 \\
\hline 3.2 & 0.5 & 0.05 & -1.5 \\
\hline 3.3 & 0.7 & 0.2 & -1 \\
\hline 3.4 & 0.5 & 0.1 & -1 \\
\hline
\end{tabular}

Table 2: Comparison of radius of convergence ball

\begin{tabular}{|c|c|c|}
\hline Examples & Method (1.2) & Method (1.3) \\
\hline 3.1 & $1.2 \times 10^{-6}$ & $4.0 \times 10^{-9}$ \\
\hline 3.2 & $6.8 \times 10^{-5}$ & $6.6 \times 10^{-5}$ \\
\hline 3.3 & 0.012069 & 0.009001 \\
\hline 3.4 & 0.001945 & 0.001801 \\
\hline
\end{tabular}

\section{Conclusions}

A fifth order iteration is used for solving nonlinear equations in Banach spaces and its semilocal and local convergence analysis is established under Hölder condition on Fréchet derivative. The existence and uniqueness theorem and error bounds for the solution are established. Both the convergence analysis are then verified on different examples including a nonlinear Hammerstein equation and convergence balls for each of them are derived. On comparing with an existing method, the larger convergence balls are obtained for all the examples.

\section{References}

[1] V. Arroyo, A. Cordero and J.R. Torregrosa; Approximation of artifical satellites' preliminary orbits: The efficiency challenge, Math. Comput. Model., Vol-54 (2011) pp. 1802-1807.

[2] A. Cordero, M.A. Hernández-Verón, N. Romero and J.R. Torregrosa; Semilocal convergence by using recurrence relations for a fifth-order method in Banach spaces, J. Comput. Appl. Math. Vol-273 (2015) pp. 205-213.

[3] I.K. Argyros, S. Hilout, Numerical methods in Nonlinear Analysis, World Scientific Publ. Comp. New Jersey 2013.

[4] I.K. Argyros, S. Hilout, M.A. Tabatabai, Mathematical Modelling with Applications in Biosciences and Engineering, Nova Publishers, New York, 2011.

[5] A. Cordero, J.A. Ezquerro, M.A. Hernández-Verón, J.R. Torregrosa, On the local convergence of a fifth-order iterative method in Banach spaces, Appl. Math. Comput. 251 (2015) 396-403.

[6] I.K Argyros, S.K.Khattri, Local convergence for a family of third order methods in Banach spaces, Journal of Mathematics, 46 (2014) 53-62.

[7] J.L. Hueso, E. Martínez, Semilocal convergence of a family of iterative methods in Banach spaces, Numer. Algorithms 67 (2014) 365-384.

[8] I.K. Argyros, A. S. Hilout, On the local convergence of fast two-step Newton-like methods for solving nonlinear equations, J. Comput. Appl. Math. 245 (2013) 1-9. 
[9] M.A. Hernández, The Newton method for operators with Holder continuous first derivative, J. Optim. Theory Appl. 109 (2001) 631-648.

[10] I.K. Argyros, S. George, Local convergence for deformed Chebyshev-type method in Banach space under weak conditions, Cogent Mathematics 2 (2015) 1-12.

[11] I.K. Argyros, S. George; Local convergence of deformed Halley method in Banach space under Holder continuity conditions, J. Nonlinear Sci. Appl. 8 (2015) 246-254.

[12] L.V. Kantorovich, G.P. Akilov, Functional Analysis, Pergamon Press, Oxford,1982.

[13] I.K. Argyros, S. George, A. A. Magreñán, Local convergence for multi-point-parametric ChebyshevHalley-type methods of higher convergence order, J. Comput. Appl. Math. 282 (2015) 215-224.

[14] I.K. Argyros, S. George; Local convergence of modified Halley-like methods with less computation of inversion, Novi Sad J. Math, Draft version.

[15] I.K. Argyros and A. A. Magreñán, A study on the local convergence and the dynamics of ChebyshevHalley-type methods free from second derivative, Numer. Algorithms DOI 10.1007/s11075-015-9981$\mathrm{x}$.

[16] S. Amat, M.A. Hernández and N. Romero; A modified Chebyshev's iterative method with at least sixth order of convergence, Appl. Math. Comput. 206 (2008) 164-174.

[17] J.A. Ezquerro, M.A. Hernández, M.A. Salanova, A Newton-like method for solving some boundary value problems, Numer. Func. Anal. Opt. 23 (2002) 791-805.

[18] P.K. Parida, D.K. Gupta, Recurrence relations for semilocal convergence of a Newton-like method in Banach spaces, J. Math. Anal. Appl. 345 (2008) 350-361.

[19] C. Chun, P. Stănicăa, B. Neta, Third-order family of methods in Banach spaces, Comput. Math. Appl. 61 (2011) 1665-1675. 